\title{
Aharonov-Bohm effect in undoped graphene: Magnetotrans- port via evanescent waves
}

\author{
M. I. KATSNELSON \\ Institute for Molecules and Materials, Radboud University Nijmegen, Heyendaalseweg 135, 6525 AJ Nijmegen, The \\ Netherlands
}

PACS 72.80.Vp - Electronic transport in graphene

PACS 73.23.Ad - Ballistic transport

PACS 03.65.Pm - Relativistic wave equations

\begin{abstract}
Using conformal mapping technique, compact and general analytic expressions for the effects of magnetic fluxes on conductance and Fano factor of undoped graphene nanoflakes in pseudodiffusive regime are derived.
\end{abstract}

Massless Dirac fermions in two dimensions have amazing property, the finite conductivity (of order of conductance quantum $e^{2} / h$ ) in the limit of zero charge carrier concentration and no disorder [1]. It can be understood in terms of transport via evanescent waves (zero modes of the Dirac operator) [2]. Despite we deal with an ideal situation (no scattering by defects) some transport properties, ' such as statistics of shot noise, are reminiscent of those for disordered metals [3] which justifies a frequently used term "pseudodiffusive regime". There are many theoretical [2-7] , and experimental $[8,9]$ works studying this regime in a context of graphene. This situation is very special. For conventional electron gas in semiconductors, in the absence of disorder, the states with definite energy (eigenstates of the Hamiltonian) can be simultaneously the states with definite current (eigenstates of the current operator) and it is the disorder that results in non-conservation of the current and finite conductivity. Contrary, for Dirac fermions the current operator does not commute with the Hamiltonian ("Zitterbewegung") which can be considered as a kind of intrinsic disorder $[2,10]$. More detailed understanding of the pseudodiffusive transport in graphene is therefore of a general interest for quantum statistical physics and physical kinetics.

Here we discuss peculiarities of the Aharonov-Bohm (AB) effect $[11,12]$ in the pseudodiffusive regime for graphene. The AB effect in graphene has been studied already theoretically [13-15] and experimentally [16, 17]. Combining a general consideration of zero-energy states for massless Dirac fermions [18] with recent elegant treatment of the transport via evanescent waves by conformal transformation technique [6] we derive simple and general rigorous formulas for any graphene flake topologically equivalent to the ring, avoiding both numerical simulations and explicit solutions of Shrödinger equation for some particular cases.

For the case of zero energy $E=0$ (undoped graphene) the Dirac equation

$$
\sigma(-i \nabla-\mathbf{A}) \psi=E \psi
$$

for the two-component spinor $\psi, \mathbf{A}$ is the vector potential and we use the units $\hbar=e=1$, splits into two independent equations for the components $\psi_{\sigma}(\sigma= \pm)$ :

$$
\left(-i \frac{\partial}{\partial x}+\sigma \frac{\partial}{\partial y}-A_{x}-i \sigma A_{y}\right) \psi_{\sigma}=0
$$

We will use the gauge $\nabla \mathbf{A}=0$ and introduce scalar magnetic potential $\varphi$ by

$$
A_{x}=\frac{\partial \varphi}{\partial y}, A_{y}=-\frac{\partial \varphi}{\partial x}
$$

thus,

$$
\nabla^{2} \varphi=-B
$$

where $B$ is the magnetic induction. The vector potential can be eliminated by a substitution [18]

$$
\psi_{\sigma}=e^{\sigma \varphi} \chi_{\sigma}
$$

Eq.(2) being satisfied for an arbitrary analytic function $\chi_{+}(z=x+i y)$ and complex conjugated analytic function $\chi_{-}(\bar{z})$. The latter can be found from boundary conditions. 
To consider the pseudodiffusive transport one can assume that the leads 1 and 2 attached to the undoped graphene flake are made from heavily doped graphene, such that the Fermi wavelength of electrons within the leads is much smaller than any geometric size of the system under consideration. Thus, the boundary conditions read $[2,3,5]$

$$
\begin{aligned}
1+r & =\psi_{+}^{(1)} \\
1-r & =\psi_{-}^{(1)} \\
t & =\psi_{+}^{(2)} \\
t & =\psi_{-}^{(2)}
\end{aligned}
$$

where $r$ and $t$ are reflection and transmission coefficients, respectively, and superscripts 1 and 2 label the boundaries attached to the corresponding leads. If the boundary of the sample is simply connected one can always choose $\varphi=0$ at the boundary and, thus, the magnetic field disappears from Eq.(6); this fact was used in Ref. [7] as a very elegant way to prove that a random vector potential does not effect on the value of minimal conductivity. Further we will consider a sample with a topology of the ring where the scalar potential $\varphi$ is still constant at each boundary but these constants, $\varphi_{1}$ and $\varphi_{2}$ are different. Also, by symmetry,

$$
\chi_{+}^{(2)} / \chi_{+}^{(1)}=\chi_{-}^{(1)} / \chi_{-}^{(2)}
$$

Further derivation follows Ref. [6] and the answer for the transmission coefficient $T=|t|^{2}$ has the form:

$$
T_{j}=\frac{1}{\cosh ^{2}[2(j+a) \ln \Lambda]}
$$

where $j= \pm 1 / 2, \pm 3 / 2, \ldots$ labels zero modes of the Dirac equation, $\Lambda$ is determined by a conformal transformation of our flake to the rectangle and

$$
a=\frac{\varphi_{2}-\varphi_{1}}{2 \ln \Lambda}
$$

For simplicity, we will consider further the case of the Corbino disc with inner radius $R_{1}$ and outer radius $R_{2}$, when [6]

$$
\Lambda=\sqrt{R_{2} / R_{1}}
$$

The conductance $G$ (per spin per valley) and Fano factor of the shot noise $F$ are expressed via the transmission coefficients (8) as

$$
\begin{aligned}
G & =\frac{e^{2}}{h} \sum_{j=-\infty}^{\infty} T_{j}, \\
F & =1-\frac{\sum_{j=-\infty}^{\infty} T_{j}^{2}}{\sum_{j=-\infty}^{\infty} T_{j}}
\end{aligned}
$$

To calculate the sums in Eq. (11) one can use the Poisson summation formula

$$
\sum_{n=-\infty}^{\infty} f(n+x)=\sum_{k=-\infty}^{\infty} \exp [-2 i \pi k x] \tilde{f}_{k}
$$

where $n, k$ are all integers and $\widetilde{f}_{k}$ is the Fourier transform of the function $f(x)$. Substituting Eq.(8) into (11) one finds a compact and general answer for the effect of magnetic field on the transport characteristics:

$$
\begin{gathered}
G=\frac{e^{2}}{h \ln \Lambda}\left[1+2 \sum_{k=1}^{\infty}(-1)^{k} \cos (2 \pi k a) \alpha_{k}\right] \\
F=1-\frac{2}{3}\left[\frac{1+2 \sum_{k=1}^{\infty}(-1)^{k} \cos (2 \pi k a) \alpha_{k}\left(1+\frac{\pi^{2} k^{2}}{4 \ln ^{2} \Lambda}\right)}{1+2 \sum_{k=1}^{\infty}(-1)^{k} \cos (2 \pi k a) \alpha_{k}}\right]
\end{gathered}
$$

where

$$
\alpha_{k}=\frac{\pi^{2} k / 2 \ln \Lambda}{\sinh \left(\pi^{2} k / 2 \ln \Lambda\right)}
$$

Eq.(4) can be solved explicitly for radially symmetric distributions of the magnetic field $B(r)$ :

$$
\varphi_{2}-\varphi_{1}=\frac{\Phi}{2 \pi} \ln \left(\frac{R_{2}}{R_{1}}\right)+\int_{R_{1}}^{R_{2}} \frac{d r}{r} \int_{0}^{r} d r^{\prime} r^{\prime} B\left(r^{\prime}\right)
$$

where $\Phi$ is the magnetic flux through the inner ring. In the case of $\mathrm{AB}$ effect where all magnetic flux is concentrated within the inner ring one has

$$
a=\frac{e \Phi}{2 \pi \hbar c}
$$

where we have restored world constants.

Due to the large factor $\pi^{2}$ in the argument of sinh in Eq. (15) only the terms with $k=1$ should be kept in Eqs.(13) and (14) for all realistic shapes, thus,

$$
\begin{gathered}
G=G_{0}\left[1-\frac{4 \pi^{2}}{\ln \left(R_{2} / R_{1}\right)} \exp \left(-\frac{\pi^{2}}{\ln \left(R_{2} / R_{1}\right)}\right) \cos \left(\frac{e \Phi}{\hbar c}\right)\right] \\
F=\frac{1}{3}+\frac{8 \pi^{4}}{3 \ln ^{3}\left(R_{2} / R_{1}\right)} \exp \left(-\frac{\pi^{2}}{\ln \left(R_{2} / R_{1}\right)}\right) \cos \left(\frac{e \Phi}{\hbar c}\right)
\end{gathered}
$$

where $G_{0}=\frac{2 e^{2}}{h \ln \left(R_{2} / R_{1}\right)}$.

Oscillating contributions to $G$ and $F$ are exponentially small for very thin rings but, for sure, measurable if the ring is thick enough. For $R_{2} / R_{1}=5$ their amplitudes are $5.3 \%$ and $40 \%$, respectively.

Pseudomagnetic fields describing by the vector potential can be created by deformations of the graphene flake [19, 20]. Expressions (13), (14) can be applied also in this situation.

Consider now a generic case with the magnetic (or pseudomagnetic [19]) field $B=0$ within the flake. Then, the solution of Eq.(4) can be obtained from the solution for the disc by the same conformal transformation which determines the function $\Lambda$. One can see immediately that Eq.(17) remains the same. As for the expressions (18), (19) they can be rewritten in terms of experimentally measurable quantity $G_{0}$,

$$
G=G_{0}\left[1-\frac{4 \pi^{2}}{\beta} \exp \left(-\pi^{2} / \beta\right) \cos \left(\frac{e \Phi}{\hbar c}\right)\right]
$$




$$
F=\frac{1}{3}+\frac{8 \pi^{4}}{3 \beta^{3}} \exp \left(-\pi^{2} / \beta\right) \cos \left(\frac{e \Phi}{\hbar c}\right)
$$

where $\beta=2 e^{2} / h G_{0}$ and we assume $\beta \ll \pi^{2}$.

To conclude, conformal transformations $[5,6]$ is a powerful tool to consider pseudodiffusive transport in undoped graphene flakes of arbitrary shape, not only without magnetic field but also in the presence of magnetic fluxes in the system. Experimental study of the Aharonov-Bohm oscillations and comparison with simple expressions (20), (21) derived here would be a suitable way to check whether the ballistic regime is reached on not in a given experimental situation.

Acknowledgements. The work is financially supported by Stichting voor Fundamenteel Onderzoek der Materie (FOM), the Netherlands. I am grateful to Pavel Ostrovsky for stimulating discussions of Ref. [7] and Carlo Beenakker for helpful critical remarks.

\section{REFERENCES}

[1] Ludwig A. W. W., Fisher M. P. A., Shankar R. and Grinstein G., Phys. Rev. B, 50 (1994) 7526.

[2] Katsnelson M. I., Eur. Phys. J., 51 (2006) 157.

[3] Tworzydlo J., Trauzettel B., Titov M., Rycerz A. and Beenakker C. W. J., Phys. Rev. Lett., 96 (2006) 246802.

[4] Prada E., San-Jose P., Wunsch B. and Guinea F., Phys. Rev. B 75 (2007) 113407.

[5] Katsnelson M. I. and Guinea F., Phys. Rev. B, 78 (2008) 075417.

[6] Rycerz A., Recher P. and Wimmer M., Phys. Rev. B 80 (2009) 125417.

[7] Schuessler A., Ostrovsky P. M., Gornyi I. V. and Mirlin A. D., Phys. Rev. B 79, 075405 (2009).

[8] Miao F., Wijeratne S., Zhang Y., Coskun U. C., Bao W. and Lau C. N., Science 317 (2007) 1530.

[9] Danneau R., Wu F., Craciun M. F., Russo S., Tomi M. Y., Salmilehto J., Morpurgo A. F. and Hakonen P. J., Phys. Rev. Lett. 100 (2008) 196802.

[10] Auslender M. and Katsnelson M. I., Phys. Rev. B 76 (2007) 235425.

[11] Aharonov Y. and Bohm D., Phys. Rev. 115 (1959) 485.

[12] Olariu S. and Popescu I. I. Rev. Mod. Phys. 57 (1985) 339.

[13] Recher P., Trauzettel B., Rycerz A., Blanter Ya. M., Beenakker C. W. J. and Morpurgo A. F., Phys. Rev. B 76 (2007) 235404.

[14] Jackiw R., Milstein A. I., Pi S.-Y. and Terekhov I. S., Phys. Rev. B 80 (2009) 033413.

[15] Wurm J., Wimmer M., Baranger H. U. and Richter K., arXiv:0904.3182 (unpublished).

[16] Russo S., Oostinga J. B., Wehenkel D., Heersche H. B., Sobhani S. S., Vandersypen L. M. K. and Morpurgo A. F., Phys. Rev. B 77 (2008) 085413.

[17] Molitor F., Huefner M., Jacobsen A., Pioda A., Stampfer C., Ensslin K. and Ihn T., arXiv:0904.3164 (unpublished).

[18] Aharonov Y. and Casher A., Phys. Rev. A, 19 (1979) 2461.

[19] Fogler M., Guinea F. and Katsnelson M. I., Phys. Rev. Lett. 101 (2008) 226804.
[20] Guinea F., Katsnelson M. I. and Geim A. K., Nature Phys. DOI:10.1038/nphys1420 (2009). 